\title{
Poiseuille Advection of Chemical Reaction Fronts
}

\author{
Boyd F. Edwards \\ ${ }^{1}$ Department of Physics, West Virginia University, Morgantown, West Virginia 26506-6315
}

(Received 29 December 2001; published 16 August 2002)

\begin{abstract}
Poiseuille flow between parallel plates alters the shapes and velocities of chemical reaction fronts. In the narrow-gap limit, the cubic reaction-diffusion-advection equation predicts a front-velocity correction equal to the gap-averaged fluid velocity $\epsilon$. In the singular wide-gap limit, the correction equals the midgap fluid velocity $3 \epsilon / 2$ when the flow is in the direction of propagation of the reaction front, and equals zero for adverse flow of any amplitude for which the front has a midgap cusp. Stationary fronts are possible only for adverse flow and finite gaps. Experiments are suggested.
\end{abstract}

DOI: 10.1103/PhysRevLett.89.104501

The convective transition for reaction fronts ascending in narrow cells has enjoyed considerable attention [1-4]. The associated dimensionless cubic reaction-diffusionadvection equation [4,5],

$$
\frac{\partial c}{\partial t}+\mathbf{v} \cdot \nabla c=\frac{1}{\eta} \nabla^{2} c+2 \eta c^{2}(1-c)
$$

accurately describes the propagation of autocatalytic iodate-arsenous acid reaction fronts, and admits an analytical soliton solution for a flat advection-free front. From left to right, the terms in Eq. (1) give the time rate of change of the catalyst concentration $c(\mathbf{x}, t)$, the rate of catalyst advection by the fluid flow, the rate of catalyst diffusion, and the cubic rate of catalyst production by the chemical reaction.

The Navier-Stokes equations demand that the 2D flow between parallel no-slip plates at $x= \pm 1$ assume the dimensionless Poiseuille velocity profile [6]

$$
\mathbf{v}(\mathbf{x}, t)=\frac{3}{2}\left(1-x^{2}\right) \overline{\mathbf{v}}(y, z, t)
$$

where $\hat{\mathbf{x}} \cdot \overline{\mathbf{v}}=0$. To simplify calculations of the convective transition, previous researchers [1,4] replace $\mathbf{v}$ by its gap average $\overline{\mathbf{v}}$ and ignore the $x$ dependence of $c$ in Eq. (1). These approximations imply a local front velocity $u=1+$ $\epsilon$ with a correction $\epsilon=\hat{\mathrm{p}} \cdot \overline{\mathbf{v}}$ equal to the dimensionless component of the average fluid velocity in the direction $\hat{p}$ of propagation of the front in the $y-z$ plane. Here, $\epsilon>0$ implies supportive Poiseuille flow in the direction of propagation of the chemical reaction front, $\epsilon<0$ implies adverse flow in the opposite direction, and $\eta$ is the ratio of the gap half-width to the reaction front thickness.

In this Letter, we show that these approximations are valid only in the $\epsilon \rightarrow 0$ and $\eta \rightarrow 0$ limits, and that $u>$ $1+\epsilon$ otherwise. Although linear calculations $[1,4]$ of the convective transition satisfy $\epsilon \rightarrow 0$, nonlinear fingering experiments [3] do not generally satisfy either $\epsilon \rightarrow 0$ or $\eta \rightarrow 0$. For the singular limit $\eta \rightarrow \infty$, we show that $u=$ $1+3 \epsilon / 2$ for $\epsilon>0$ and that $u=1$ for $\epsilon<0$, indicating that adverse Poiseuille flow through wide gaps has no effect whatever on the front velocity, although such flow
PACS numbers: 47.70.Fw, 05.45.Yv, 82.40.Ck, 83.10.Bb

stretches the front significantly and endows it with a midgap cusp. This unexpected behavior should be observable in experiments and may be observable for other types of propagating fronts, such as premixed flames.

The chemical reaction produces its own catalyst, whose molecular diffusion into the unreacted fluid limits the speed of propagation of the reaction front, which is typically $[4,5] U_{0} \approx 0.03 \mathrm{~mm} / \mathrm{s}$ for a flat advection-free front. The catalyst concentration increases monotonically from its value of zero far ahead of the propagating front to its value $C_{2}$ far behind, and increases steeply within a reaction front of typical thickness $L_{0}=D_{C} / U_{0} \approx 0.07 \mathrm{~mm}$. Here, $D_{C}$ is the catalyst molecular diffusivity. In Eqs. (1) and (2), lengths are scaled by the gap half-width $a / 2$, velocities are scaled by $U_{0}$, concentrations are scaled by $C_{2}$, and $\eta=a / 2 L_{0}$.

We seek soliton solutions of Eq. (1) propagating in the $\hat{\mathrm{p}}=\hat{\mathrm{z}}$ direction without change of shape at a constant velocity $u$, for steady Poiseuille flow with $\epsilon=$ const. Such solutions satisfy

$$
\frac{1}{\eta^{2}} \frac{\partial^{2} c}{\partial x^{2}}+\frac{\partial^{2} c}{\partial \zeta^{2}}+\left[u-\frac{3}{2} \epsilon\left(1-x^{2}\right)\right] \frac{\partial c}{\partial \zeta}+2 c^{2}(1-c)=0,
$$

where the concentration $c(x, \zeta)$ now depends on a convenient comoving coordinate $\zeta=\eta(z-u t)$, and satisfy the boundary conditions $c(x,+\infty)=0, c(x,-\infty)=1$, and $\partial c( \pm 1, \zeta) / \partial x=0$.

The limit $\epsilon \rightarrow 0$ of small-amplitude flows is accessible to elementary perturbation theory. Inserting expansions $c=c_{0}+c_{1}+\ldots$ and $u=u_{0}+u_{1}+\ldots$ in powers of $\epsilon$ into Eq. (3) gives $u=1+\epsilon$ and $c(x, z, t)=\{1+$ $\left.e^{\eta[z-u t-h(x)]}\right\}^{-1}$ through first order [7]. Here, $z=h(x)+$ $u t$ locates the surface of concentration $c=1 / 2$ at the center of the reaction front, whose shape is given by

$$
h(x)=\frac{\epsilon \eta}{8}\left(1-x^{2}\right)^{2} .
$$

The narrow-gap limit $\eta \rightarrow 0$ can be studied by expanding in even powers of $\eta$ according to $c=c^{(0)}+c^{(2)}+\ldots$ 
and $u=u^{(0)}+u^{(2)}+\ldots$. Through zeroth order, Eq. (3) gives $u=1+\epsilon$, as before, and $c(z, t)=\left[1+e^{\eta(z-u t)}\right]^{-1}$. Accordingly, for gap widths that are small compared with the front width, molecular diffusion precludes any front curvature and averages the fluid contributions to the front velocity, yielding a front-velocity enhancement equal to the average fluid velocity $\epsilon$.

In the wide-gap limit $\eta \rightarrow \infty$, the shape $h(x)$ of the vanishingly thin front satisfies an ordinary differential "eikonal" equation

$$
u=\sqrt{1+\left(h^{\prime}\right)^{2}}+\frac{3}{2} \epsilon\left(1-x^{2}\right)+\frac{1}{\eta}\left[1+\left(h^{\prime}\right)^{2}\right]^{-1} h^{\prime \prime},
$$

which is derivable [8-10] from Eq. (1). Solving Eq. (5) requires care because the coefficient of its second-order curvature term vanishes as $\eta \rightarrow \infty$. Ignoring this term leaves a first-order equation

$$
u=\sqrt{1+\left(h^{\prime}\right)^{2}}+\frac{3}{2} \epsilon\left(1-x^{2}\right),
$$

which is valid except near singular regions where $h^{\prime \prime}$ is of order $\eta$. Since $h(-x)=h(x)$, we can confine our attention

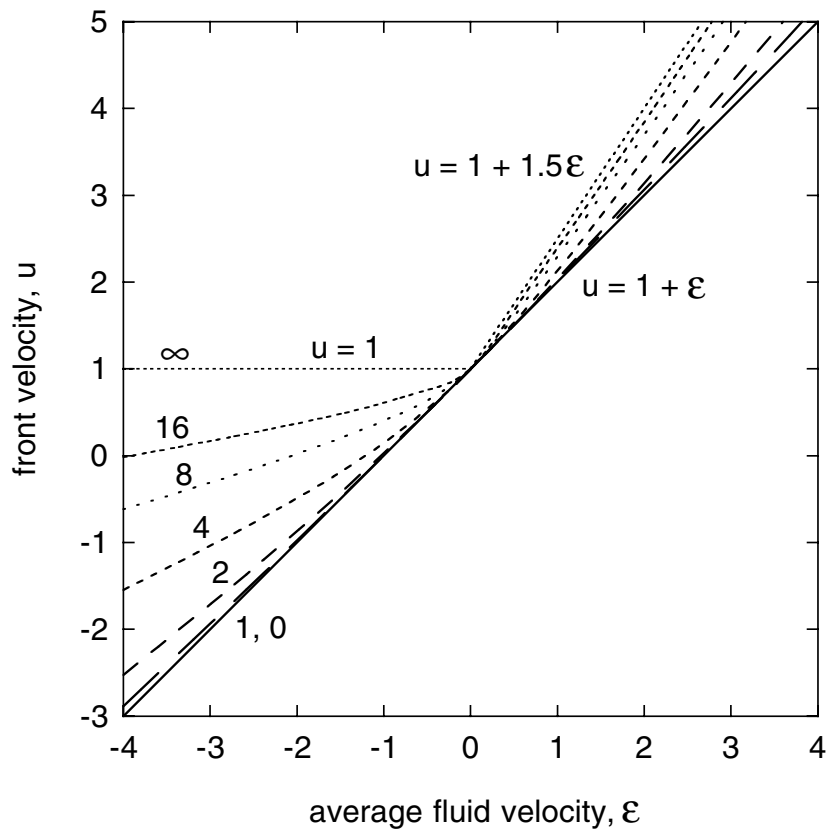

FIG. 1. Dimensionless reaction front velocity $u$ vs the dimensionless average velocity $\epsilon$ of the Poiseuille flow between parallel plates, for various ratios $\eta$ of the gap half-width to the front thickness. The front velocity increases monotonically with increasing $\eta$ at fixed $\epsilon$, and is bounded from below by the $\eta \rightarrow 0$ result $u=1+\epsilon$ (solid trace) and from above by the singular $\eta \rightarrow \infty$ results $u=1$ for $\epsilon<0$ and $u=1+3 \epsilon / 2$ for $\epsilon>0$ (shortest dashes). Each trace for finite $\eta$ has continuous slope, and matches the value and slope of the $\epsilon \rightarrow 0$ result $u=1+\epsilon$ near $\epsilon=0$. Stationary fronts and reversed-direction fronts with $u=0$ and $u<0$ are possible only for adverse flow satisfying $\epsilon \leq-1$. Results for $\eta \rightarrow 0, \eta \rightarrow \infty$, and $\epsilon \rightarrow 0$ were obtained analytically, and all other results were obtained numerically. to the interval $0 \leq x \leq 1$, with the boundary conditions $h^{\prime}(0)=h^{\prime}(1)=0$. Only one of these conditions can be satisfied by choosing the eigenvalue $u$ in Eq. (6), with the sign of $\epsilon$ determining which one. To satisfy the other condition requires a singular boundary-layer solution [7] whose value and slope match the solution given by Eq. (6) at the edge of the boundary layer. This boundary layer is ignored herein because its thickness vanishes as $\eta^{-1}$, and because $u$ can be obtained without it. For $\epsilon>0$, Eq. (6) satisfies only $h^{\prime}(0)=0$ because $\left|h^{\prime}\right|$ increases with increasing $x$, whence $u=1+3 \epsilon / 2$. For $\epsilon<0$, Eq. (6) satisfies only $h^{\prime}(1)=0$ because $\left|h^{\prime}\right|$ decreases with increasing $x$, whence $u=1$.

To explore the behavior for general $\epsilon$ and $\eta$, we also solve Eq. (1) numerically using finite differences [7], allowing the transients to decay away sufficiently before identifying the steady soliton solutions.

Figure 1 summarizes the front-velocity results, which are confined to a double wedge centered on the velocity $u=1$ of a flat advection-free reaction front with $\epsilon=0$. Remarkably, the velocity $u=1$ persists for arbitrary adverse flow amplitudes $\epsilon<0$ in the wide-gap limit $\eta \rightarrow \infty$. Accordingly, contrary to expectation, adverse Poiseuille flow of any amplitude through wide gaps is incapable of slowing the front or of reversing its direction of propagation. For gaps of finite width, adverse flow can indeed slow or reverse the front; the intersections of the various traces in Fig. 1 with the $u=0$ line determine the paired values of $\epsilon$ and $\eta$ which give stationary fronts. Such reversals occur when significant lateral diffusion limits the front distortion.

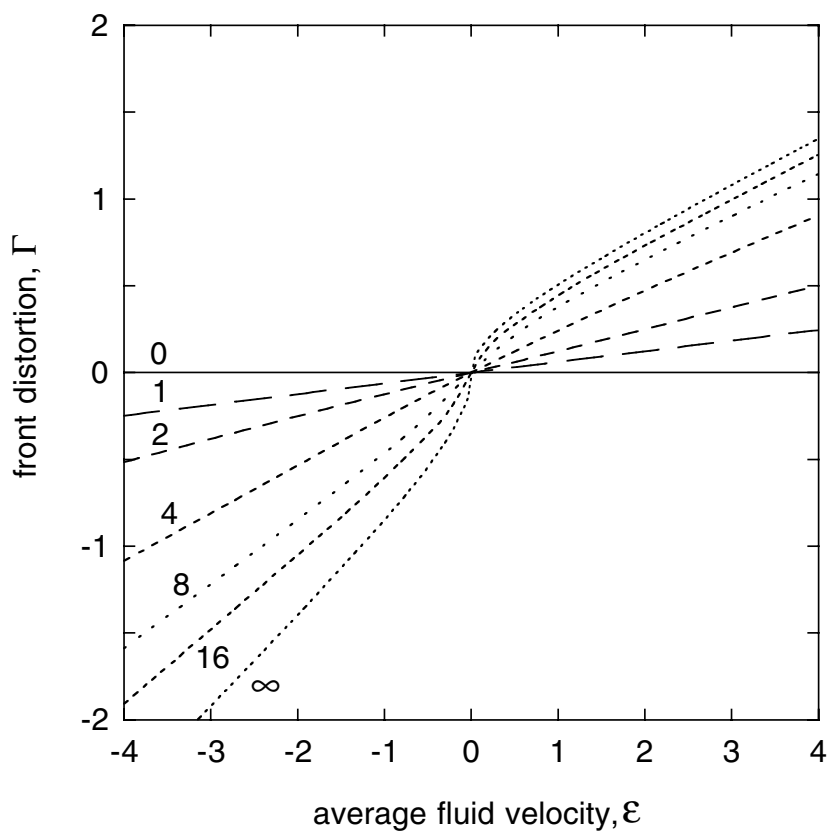

FIG. 2. Front distortion $\Gamma$ vs $\epsilon$, for various values of $\eta$. Results for $\eta \rightarrow \infty$ (shortest dashes) are obtained by integrating Eq. (6). Numerical results for finite $\eta$ (longer dashes) evidently match the analytical result $\Gamma=\epsilon \eta / 16$ for small $\eta$ and finite $\epsilon$, and for small $\epsilon$ and finite $\eta$. 
Supportive Poiseuille flow with $\epsilon>0$ through wide gaps increases the front velocity by an amount equal to the midgap fluid velocity $3 \epsilon / 2$. Thus, in contrast with largeamplitude adverse flows through wide gaps, large-

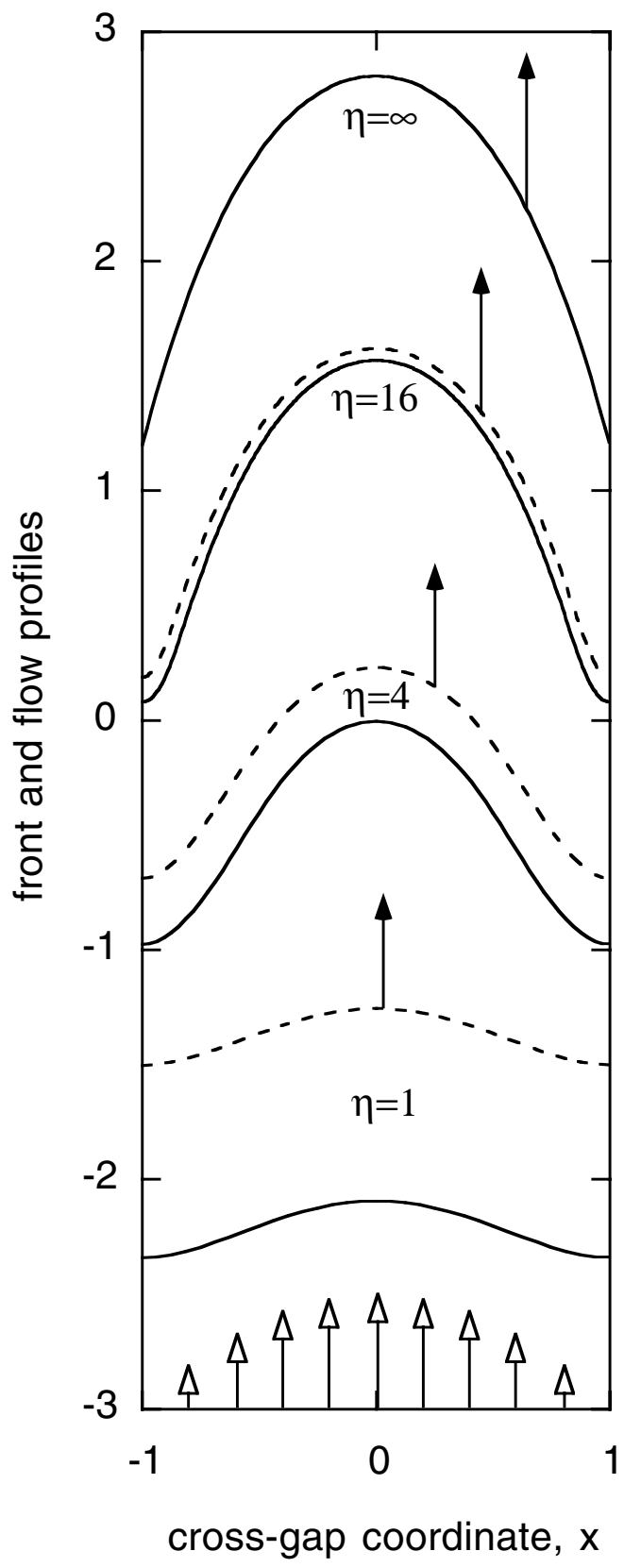

FIG. 3. Front and flow profiles for supportive Poiseuille flow with $\epsilon=2$, for which the flow is in the same direction as the chemical reaction, with parallel plates located at $x= \pm 1$. For each value of $\eta=1,4,16$, and $\infty$, a pair of constantconcentration traces with catalyst concentrations $c=2 / 5$ (dotted trace, reaction $40 \%$ completed) and $c=3 / 5$ (solid trace, reaction $60 \%$ completed) is shown. The separation between these traces is comparable to the front thickness, which vanishes for $\eta \rightarrow \infty$. Arrows with solid heads represent the front velocities $u=3.04,3.42,3.85$, and 4.00 for $\eta=1,4,16$, and $\infty$. Arrows with hollow heads represent the quadratic Poiseuille flow. The $\eta \rightarrow \infty$ trace was obtained by integrating Eq. (6). amplitude supportive flows through wide gaps produce large reaction front velocities.

To measure the distortion of the reaction front by the flow, we define $\Gamma=[h(0)-h( \pm 1)] / 2$ as the ratio of the height of the $c=1 / 2$ front profile to the gap width. The result $\Gamma=\epsilon \eta / 16$ obtained from Eq. (4) matches our

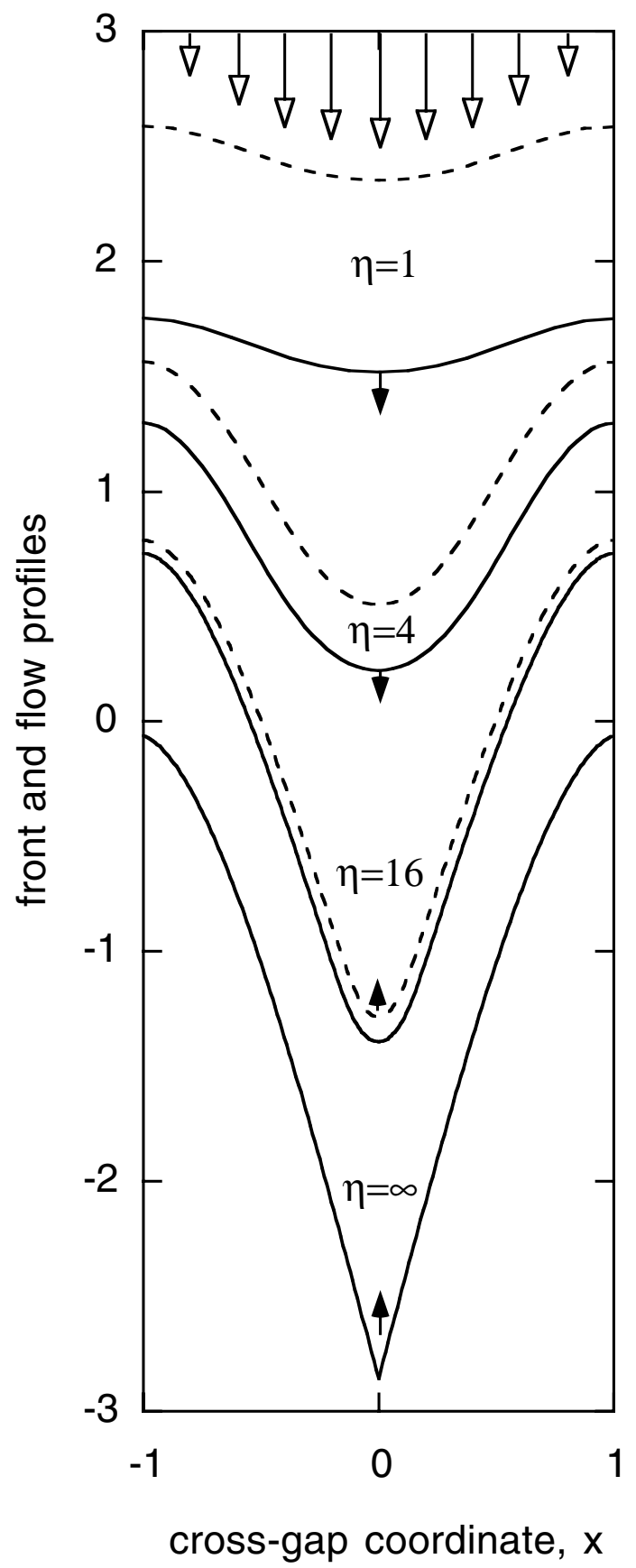

FIG. 4. Front and flow profiles for adverse Poiseuille flow with $\epsilon=-2$, similar to Fig. 3. The front velocities $u=-0.98$, $-0.49,0.37$, and 1.00 for $\eta=1,4,16$, and $\infty$ indicate that the flow succeeds in reversing the direction of propagation of the reaction front only for narrow gaps. In the wide-gap limit $\eta \rightarrow$ $\infty$, the singularity of Eq. (5) demands a trailing midgap cusp in the front, which propagates at its advection-free velocity $u=1$. The $\eta \rightarrow \infty$ trace was obtained by integrating Eq. (6). 
numerical results for $\Gamma$ vs $\epsilon$ (Fig. 2) near $\epsilon=0$, and for small $\eta$ and finite $\epsilon$. This latter result can be confirmed analytically by carrying the small- $\boldsymbol{\eta}$ expansion to second order [7], which reproduces the front shape given by Eq. (4). Evidently, $\Gamma$ and $\epsilon$ always share the same sign, indicating that the midgap region of the reaction front bulges in the direction of the Poiseuille flow. Furthermore, $|\Gamma|$ increases with both $\eta$ and $|\epsilon|$.

Figures 3 and 4 show the front profiles for $c=2 / 5$ (dotted traces) and $c=3 / 5$ (solid traces), for $\eta=1,4$, 16 , and $\infty$. For each value of $\eta$, these profiles bracket the steeply varying central region of the reaction front, of thickness comparable to the front thickness $L_{0}$ in conventional units. The chemical reaction consumes unreacted fluid ahead of the $c=2 / 5$ profile to produce reacted fluid behind the $c=3 / 5$ profile. For $\eta \rightarrow \infty$, these profiles coincide because the front is thin compared with the gap. In Fig. 3, the supportive Poiseuille flow is in the direction of propagation of the chemical reaction front, whereas the flow is adverse in Fig. 4, leading to significant differences in the front profiles. Adverse flow stretches the front markedly in the direction of the flow, and endows it with a midgap cusp in the wide-gap limit $\eta \rightarrow \infty$. This stretching increases the surface area of the front, allowing it to consume more fluid in order to avoid being slowed down by the flow.

Replacing $\mathbf{v}$ by its gap average and ignoring the $x$ dependence of $c$ in Eq. (1) are appropriate both for small $\epsilon$ and for small $\eta$; both give $u=1+\epsilon$. These approximations should therefore be valid near the critical point for the onset of buoyancy-driven convection [1,4], where the convective amplitude $\epsilon$ is small. However, $u=1+\epsilon$ underestimates the front velocity for finite-amplitude flows relevant to nonlinear fingering, and must be replaced by the results $u(\epsilon, \eta)$ given in Fig. 1. For this purpose, we have constructed an empirical formula for $u(\epsilon, \eta)$ (Ref. [7]) which matches the numerical data to within a few percent over the entire computed range and which exactly reproduces the limiting values.

The unexpected refusal of reaction fronts to be slowed by wide-gap adverse Poiseuille flow and the appearance of the associated midgap cusp in the reaction front should be easily observable in experiments on propagating autocatalytic reaction fronts. Since such behavior arises directly from the insignificance of front curvature except very near the cusp, this behavior might also be observable for pre- mixed flames and for other systems involving propagating fronts, when the gap width is large compared with the front thickness. Experiments would be especially straightforward for propagation through cylindrical capillary tubes, for which calculations are underway. A wide parameter range is accessible experimentally, by adjusting the dimensionless flow rate $\epsilon$ and gap width $\eta$. The $0.01 \%$ difference [9] between the mass densities of the unreacted and reacted fluids suggests that experiments be carried out with the reaction proceeding downward, with the unreacted fluid below the less dense reacted fluid, to avoid buoyancy effects. Even under these conditions, distortion of the front raises the overall gravitational potential energy above the flat-front value and might lead to small buoyancy-driven convection currents which would likely reduce the distortion and velocity of the front. Whether such currents would destabilize the cusp is an open question. Although it might be difficult to observe such currents directly, they should be accessible theoretically, using the Navier-Stokes equations augmented to include small changes in the fluid density.

Helpful interactions with M. Böckmann, A. De Wit, W. F. Edwards, E. Reynolds, K. Showalter, R. Spangler, D. Vasquez, and J. Wilder are gratefully acknowledged.

[1] A. De Wit, Phys. Rev. Lett. 87, 054502 (2001).

[2] M. Böckmann and S. C. Müller, Phys. Rev. Lett. 85, 2506 (2000).

[3] M. R. Carey, S. W. Morris, and P. Kolodner, Phys. Rev. E 53, 6012 (1996).

[4] J. Huang and B. F. Edwards, Phys. Rev. E 54, 2620 (1996).

[5] A. Saul and K. Showalter, in Oscillations and Traveling Waves in Chemical Systems, edited by R. J. Field and M. Burger (Wiley, New York, 1985), p. 419; A. Hanna, A. Saul, and K. Showalter, J. Am. Chem. Soc. 104, 3838 (1982).

[6] L. D. Landau and E. M. Lifshitz, Course of Theoretical Physics: Fluid Mechanics (Pergamon, New York, 1987), Chap. II.

[7] B. F. Edwards (to be published).

[8] J. J. Tyson and J.P. Keener, Physica (Amsterdam) 32D, 327 (1988).

[9] B. F. Edwards, J. W. Wilder, and K. Showalter, Phys. Rev. A 43, 749 (1991).

[10] B. F. Edwards and R. Spangler (to be published). 